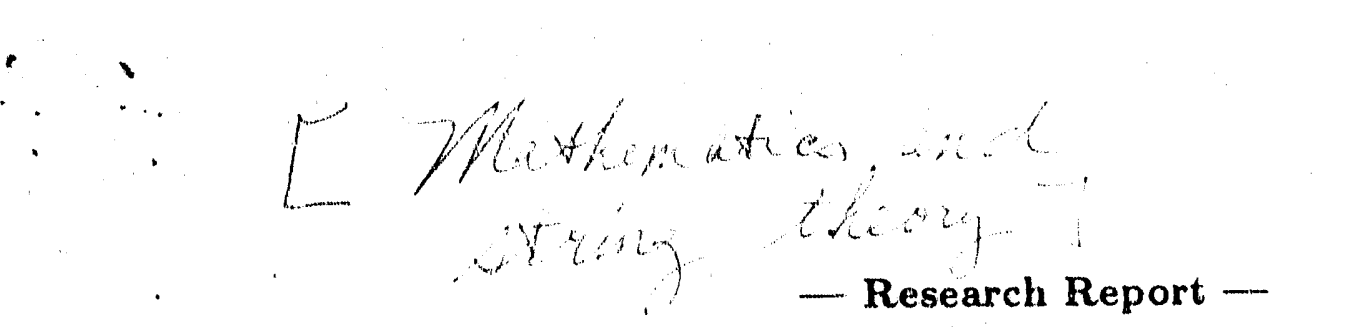

Over the past year our research activities concentrated around: (1) non-commutative differential geometry and its connections with quantum physics and (2) 2-dimensional (super)conformal quantum field theories and related non-linear $\sigma$-models.

\title{
- Part 1 -
}

Non-commutative differential geometry, whose foundations were laid by A. Connes, is a new area of mathematical research with a big potential for development. A natural field of application for the ideas of non-commutative geometry is the study of geometric structures arising from quantum physics. A natural tool to study these is the Pfaffian on infinite dimensional spaces [JL]. Quantization of classical spaces leads to examples of both finite ( $p$-summable) and infinite dimensional ( $\Theta$-summable) non-commutative (quantum) spaces.

A quantum space is defined in terms of a non-commutative $C^{*}$-algebra $\mathcal{A}$ which can be thought of as the "algebra of continuous functions" on a non-commutative space. This algebra involves a deformation parameter, Planck's constant (or, more generally, a space of such parameters). One requires that a suitable limit of this algebra exist, as the deformation parameter approaches zero. One, in effect, studies the "semi-classical limit" of the non-commutative algebra. The key relation of this limit is that the commutator of two "quantized functions" approaches zero, with a first order correction given by the Poisson bracket on the manifold.

In Refs. [KL4] and [KL5], Lesniewski and Klimek initiated a program of constructing and studying quantum deformations of hyperbolic manifolds. They constructed $C^{*}$ algebras of continuous functions on the unit disc and hyperbolic compact Riemann surfaces. The central concept of this construction is that of a Toeplitz operator on a suitable Bergman space. They established theorems describing the structure of these $C^{*}$-algebras prove deformation estimates. In Ref. [KL6] this construction was extended to a two-parameter deformation of the unit disc, the second parameter being related to the parameter $q$ which appears in the theory of quantum groups. The resulting $C^{*}$-algebras have a rich structure which is related to the classification of $S U_{q}(1,1)$-covariant Poisson structures on the unit disc.

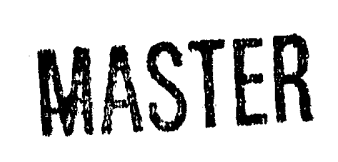


In Ref. [BLU], Borthwick, Lesniewski and Upmeier extended some of the results of Ref. [KL4] to the general setup of (both classical and exceptional) Cartan domains. This gives rise to an infinite sequence of non-commutative spaces. They constructed the corresponding deformed $C^{*}$-algebras of continuous functions and established the deformation estimates. The project of studying these spaces is currently in progress, and we hope to get more insight into the structure of these non-trivial quantum spaces.

The ultimate goal of this line of development is to study topological invariants of the quantum spaces. The relevant framework was proposed by $A$. Connes arid involves the notion of cyclic cohomology (a non-commutative extension of de Rham cohornology) and a Fredholm module (a non-cornmutative version of metric structure of a manifold). In a work by Jaffe, Lesniewski and Osterwalder, an intimate connection between the notion of a Fredholm module and the physics concept of supersymmetry was emphasized. This connection leads to a natural construction of a fundamental cohomology class in non-commutative geometry, the Chern character. The work [BKLR2] contains a construction of Fredholm modules over quantized Cartan domains and flat spaces as well as the computation of the Chern character (in the JLO framework). The construction relies on the concepts of quantized supermanifolds and super Toeplitz operators [BKLR1]. It turns out that, in all the examples which we have studied, there is an underlying $N=2$ supersymmetry, whose generators serve as the Dirac operators of the relevant Fredholm modules.

In another work [LR], the structure of the set of *-representations of the $C^{*}$-algebra of continuous functions on the quantum group $S U_{q}(2)$ was studied. It is shown that a tensor product of two such representations can be decomposed into a direct integral of irreducible representations. In [FT], the Hochschild and cyclic homologies of a large class of quantum groups were cornputed. The concept of a quantum group was also the subject of study in Refs. [Z1] and [Z2]. The papers [KKL] and [KL3] contain an equivariant extension of the framework of entire cyclic cohomology.

\section{- Part $2-$}

Axelrod studied the perturbation theory for Chern-Simons quantum field theory and its generalizations [AS]. This project has been ongoing since the spring of 1991, in collaboration with I.M. Singer. The first stage of this work specializes to perturbing about an acyclic flat connection on a closed three manifold $M$. It is proved that the theory is well-defined 
and finite and that, after subtracting a "counterterm" in exactly the manner expected physically, the terms in the perturbation theory define topological invariants. The striking simplicity of this proof arises from the observation that the gauge fixed action can be given a superspace formulation. Mathematically, this translates into the fact that the Feynman rules describing the perturbation theory all have simple interpretations in terms of differential forms. The results follow then from a few basic properties of the propagator $L$, including a geometrical description of the propagator singularities in terms of equivariarit cohomology.

Currently, these results are being generalized in several different ways allowing for arbitrary dimensional manifolds and non-acyclic connections, using "coupling constants" more general than Lie algebra structure constants, incorporating links, and making explicit the connection to moduli spaces of complex curves. To this end, the original propagator and the derivation of its crucial properties has been greatly refined and several methods for dealing with the singularities of the Feynman integrands have been introduced. These generalizations arise naturally from the physical point of view and are closely connected with a topological open string field theory being considered by $\mathrm{E}$. Witten. They are also part of a long range program of reproducing the results of Witten's exact solution of Chern-Simons theory via perturbation theory.

The study of Dirac operators has provided an extremely fruitful interface between Physics and Mathematics for the better part of this century. Following E. Witten's geometric interpretation of elliptic genera, Liu constructs [L1] mod-2 elliptic genera on m. nifolds of dimensions 1 and $2(\bmod 8)$, obtains an integral formula for the mod- 2 index of the Dirac operator and finds topological obstructions for groups actions as a by-product. He also finds characteristic series for the Witten genera by Jacobi theta-functions and proves that Krichever's genera can be written as indices of certain twisted Dirac operators. In another article [L2], Liu gives a simple algebro-geometric proof of the so-called 'Witten rigidity theorems', which are loop-space analogues of the rigidity of the indices of classical complexes (the Euler characteristic, the signature and the $\hat{A}$-genus).

2-dimension (super)conformal quantum field theories received much attention recently as the underlying theory of (super)string propagation. However, they are also interesting by being relatively simple as quantum field theories and so providing workable paradigms that serve as guidance in the general case. Indeed, in Ref. [M], Mathur studied integrable deformations of rational conformal field theories. While the conformal field theories are 
characterized by quantum algebras (and are indeed also used in studying the latter), their integrable to deformations correspond quantum affine (i.e., Kac-Moody) algebras. That is, each term in a perturbation series around a conformal field theory decomposes into products of holomorphic and antiholomorphic "blocks", which in turn provide an affine extension of the $s u_{q}(2)$ algebra of the initial conformal field theory. A class of primary fields then corresponds to level- $k$ representations of $\widehat{s u}_{q}(2)$, with $q=e^{2 i \pi p /(k+2)}, p$ an integer.

Continuing the study of (2,2)-supersymmetric superstring compactification, Berglund, Greene and Hübsch [BGH] have uncovered a previously unsuspected degree of correlation between the classical description, in therms of a Calabi-Yau manifold, and the quantum theory in terms of a Landau-Ginzburg orbifold. The latter models are defined as effective 2-dimensional field theories and are analyzed with the aid of semiclassical field theory methods pioneered by $\mathrm{E}$. Witten a decade ago and developed recently by $\mathrm{C}$. Vafa. The corresponding Calabi-Yau algebraic varieties lend themselves to the application of equivariant cohomological algebra, which manifests certain cohomological obstructions in general. In the Landau-Ginzburg chiral ring, these show up as nontrivial contributions from twisted sectors. However, beyond the expected final agreement between these two approaches, a detailed and direct correspondence between the analysis of each is found. Furthermore, this shows that physical reasoning based upon spectral flow arguments for determining the spectrum of Landau-Ginzburg orbifolds finds direct mathematical justification in Koszul complex calculations and also that careful point-field analysis continues to recover surprisingly much of the "stringy" features.

Finally, there emerge two a priori unrelated ring structures: that defined by the Koszul complex computation in the classical analysis and the so-called chiral ring structure defined for the Landau-Ginzburg orbifolds. Ref. [BGH] shows that these two ring structures are in fact identical. The relation of this ring structure to the "physical" one, defined in the superconformal limit of the renormalization flow, remains an extremely important open issue and is being addressed in Ref. [BH2].

$(2,2)$-supersymmetric 2-dimensional field theories occur in practically identical pairs, leading to physically equivalent superstring compactifications. On the other hand, the geometric interpretation of such two models via superstring propagation through CalabiYau manifolds requires a so-called mirror pair of (at least) two topologically distinct Calabi-Yau manifolds. Until recently, explicit constructions of such mirror manifolds were 
known only for local deformations of Fermat-type (weighted) hypersurfaces. Berglund and Hübsch [BH1] have generalized this to a rather larger collection of 16 classes of nondegenerate weighted hypersurfaces and their orbifolds and have proven the mirror property using semiclassical field theory techniques of Landau-Ginzburg orbifolds.

Continuing in part an earlier work under this grant by Greene, Shapere, Vafa and Yau, in Ref. [GH], Green and Hübsch study the possibility of spacetime viriation of the compactification vacuum. The requirement of (complex) analyticity of such variations implies in general that the compactification space must singularize at certain loci in spacetime while leaving the total (10-dimensional) spacetime perfectly smooth. The singular locus assumes the shape of the world sheet of : (set of) cosmic string(s). Furthermore, the Euler characteristic of this singular locus is a topological invariant and (a) in the static case counts the cosmic strings while (b) in the dynamical case counts the number of self-interaction of this cosmic closed string. The presence of these cosmic strings curves spacetime and induces a natural spacetime metric which turns out to be a suitably gauge-fixed form of the Kähler potential of the Zamolodchikov metric. A number of observable local data about these cosmic strings is readily calculable for any particular model.

The mirror manifold conjecture has a number of mathematically rather unsuspected corollaries; as a conclusive proof of the conjecture still eludes us, proving some of the corollaries appears interesting in its own right. Also, such circumstantial data is bound to specify the mirror manifold conjecture in more detail and hopefully provide the desired insight leading to its proof. For $\mathcal{M}$ and $\mathcal{W}$ a pair of mirror manifolds, $\oplus_{q} H^{q, q}(\mathcal{M})$ and $\oplus_{q} H^{3-q, q}(\mathcal{W})$ can be given identical ring structures. It is elementary that the former of these admits an $S L(2)$ action generated by multiplication with the Kähler form. The existence of such an action in $\oplus_{q} H^{3-q, q}$ is far from even plausible. Hübsch and Yau [HY1] find such an action in $\oplus_{q} H^{3-q, q}(\mathcal{W})$ when $\mathcal{W}$ is a weighted hypersurface by explicitly constructing the action on the Jacobian ring of the hypersurface, to which $\oplus_{q} H^{3-q, q}$ is canonically isomorphic.

A formally precise and detailed description of (2,2)-superconformal 2-dimensional quantum field theories is attempted in Ref. [HY2]. We aim at a definitive understanding of the mirror manifold conjecture and superconformal field theories per se. This comprehensive review should hopefully also bring this recently developed area in theoretical physics closer to mathematicians. 
Most of his time and effort, however, Hübsch has devoted to finishing his book on Calabi-Yau manifolds [H], which is meant to abridge the gap between the existing "physics" references on differential and algebraic geometry and advanced texts on algebraic geometry such as the classic volume by Griffiths and Harris, for example. The introductory chapter presents the topic from a physicist's point of view and specifies the desired details of CalabiYau compactification. The next 14 chapters present techniques for construction of CalabiYau manifolds, the analysis of their relevant cohomology and a preliminary study of the incomplete but rapidly developing theory of Calabi-Yau moduli spaces. The last chapter then returns to some recent developments in the corresponding 2 -dimensional quantum field theory. In addition, the book contains a Lexicon where $150+$ terms and ideas are given a 1-2 paragraph brief description/definition and which may be used independently of the main part as a quick reference.

In conclusion, we have embarked on several fascinating projects and somewhat unexpectedly uncovered some new avenues for further research. We believe that they open up new fields of research and plan to continue our work in these directions, as indicated above.

\title{
DISCLAIMER
}

\begin{abstract}
This report was prepared as an account of work sponsored by 2.1 agency of the United States Government. Neither the United States Government nor any a yency thereof, nor any of their employees, makes any warranty, express or implied, or assumes iny legal liability or responsibility for the accuracy, completeness, or usefulness of any information, apparatus, pioduct, or process disclosed, or represents that its use would not infringe privately owned rights. Reference herein to any specific commercial product, process, or service by trade name, trademark, manufacturer, or otherwise does not necessarily constitute or imply its endorsement, recommendation, or favoring by the United States Government or any agency thereof. The views and opinions of authors expressed herein do not nesessarily state or reflect those of the United States Government or any agency thereof.
\end{abstract}



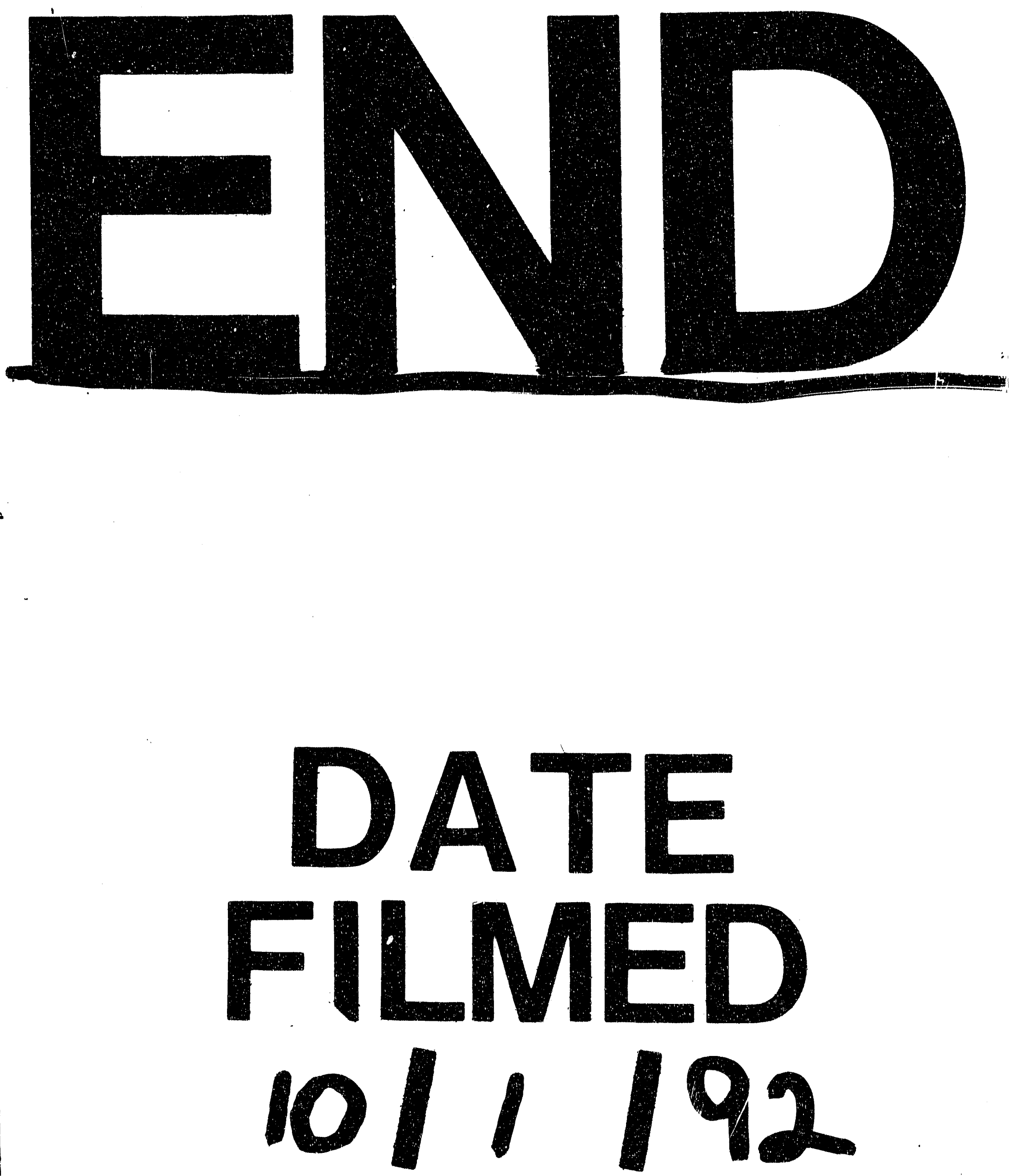
